\title{
O EFEITO DE DIFERENTES NÍVEIS DE PH EM SOLUÇÕES DE FLÚOR A 1,1\% NA CORROSÃO DE ARCOS NITI
}

\section{THE EFFECT OF DIFFERENT PH LEVELS ON FLUORIDE SOLUTIONS AT 1.1\% ON CORROSION OF NITI ARCHS}

\author{
Fernando Akio Maeda* \\ Nívea Amaral* \\ Celso Massahiro Ogawa** \\ Saulo Aguiar Farias \\ Karin de Sá Fernandes"**** \\ Paulo Eduardo Guedes Carvalho" \\ Tarcila Triviño \\ Lucas Hian da Silva
}

\begin{abstract}
RESUMO
Objetivo: Determinar o pH crítico para ocorrer a corrosão de arcos NiTi em soluções de flúor a 1,1\%, variando o pH de 3,5 a 7,0. Materiais e Métodos: Doze amostras com 10mm de comprimento de fio superelástico $\mathrm{NiTi} 0017$ x 0025 (Abzil), obtidas do segmento distal dos arcos. As amostras foram aleatoriamente separadas e imersas dentro de recipientes contendo $40 \mathrm{ml}$ de seis soluções (água, soluções fluoretadas com pH: 3,5; 4,0; 4,$5 ; 5,0 ; 5,5 ; 6,0 ; 6,5$ e 7,0), sob uma mesa agitadora durante 90 minutos. Todas as amostras foram, então, levadas ao MEV. As imagens das amostras imersas em água e soluções fluoretadas com pH 7,0, 6,5 e 6,0 não se apresentaram diferentes, no entanto a partir da solução fluoretada com $\mathrm{pH} 5,5$, as imagens superficiais apresentaram características diferentes dos grupos anteriores. Conclusão: Com base nos resultados obtidos neste estudo, o pH crítico para corrosão de arcos NiTi em soluções de flúor a 1,1\% parece estar entre 6,0 e 5,5.

Descritores: Arco NiTi · Corrosão • Solução Fluoretada
\end{abstract}

\section{ABSTRACT}

Objective: To determine the critical $\mathrm{pH}$ to occur corrosion of $\mathrm{NiTi}$ arches in $1.1 \%$ fluoride solutions by varying the $\mathrm{pH}$ between 3.5 and 7.0. Materials and Methods: Twelve samples of $10 \mathrm{~mm}$ length superelastic NiTi wire $0017 \times 0025$ (Abzil) obtained in the distal segment of the arcs. The samples were randomly separated and immersed in $40 \mathrm{ml}$ of six solutions (water, fluoride solutions with $\mathrm{pH}: 3.5 ; 4.0 ; 4.5 ; 5.0 ; 5.5 ; 6.0 ; 6.5$ and 7.0) under a shaker table for 90 minutes. All samples were observed under SEM. The images of the samples immersed in water and fluoride solutions at $\mathrm{pH}$ 7.0, 6.5 and 6.0 did not show differences, however from the fluoride solution at $\mathrm{pH} 5.5$, the surface characteristics of images presented differences from above groups. Conclusion: Based on the results obtained in this study the critical $\mathrm{pH}$ for corrosion of $\mathrm{NiTi}$ arches in fluoride solutions to $1.1 \%$ appears to be between 6.0 and 5.5.

Descriptors: NiTi arch · Corrosion • Fluoride solutions.

\footnotetext{
* Universidade Cidade de São Paulo - Professor, fernandoakiomaeda@gmail.com

** Universidade Cidade de São Paulo - Graduanda, niveaa amaral@hotmail.com

*** Universidade Paulista - Mestre, celsomassahiro@gmail.com

**** Universidade Cidade de São Paulo - Mestrando, sauloaguiar88@hotmail.com

***** Universidade Cidade de São Paulo - Professor, karin.fernandes@unicid.edu.br

****** Universidade Cidade de São Paulo - Professor, paulo@carvalho-ortodontia.com.br

******* Universidade Cidade de São Paulo - Professor, tarcilatrivino@uol.com.br

******** Universidade Cidade de São Paulo - Professor, cdhian@gmail.com
} 
MAEDA FA

AMARAL N

OGAWA CM

FARIAS SA

FERNANDES KS

CARVALHO PEG

TRIVIÑO T

SILVA LH

O EFEITO DE DIFERENTES

NÍVEIS DE PH

EM SOLUÇÕES DE

FLUUOR A $1,1 \%$

NA CORROSÃO DE ARCOS NITI

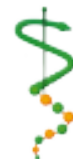

REV, ODONTOL.

UNIV, CID, SÃO

PAULO

2017; 29(3): 237-42, SET-DEZ

\section{N T RO DUÇÃO}

Os sistemas arcos-braquetes são muito utilizados na ortodontia com objetivo de se obter a movimentação dentária ${ }^{1}$. Para que essa movimentação seja mais eficaz, eficiente e fisiológica durante as fases iniciais de alinhamento e nivelamento, esse sistema deve gerar baixas forças ortodônticas, contínuas e dentro dos limites fisiológicos ${ }^{2,3}$. Nesse intuito, as ligas de níquel titânio (NiTi) têm sido amplamente utilizadas graças à biocompatibilidade e às suas propriedades mecânicas como: efeito de memória da forma e superelasticidade ${ }^{4}$. As ligas de $\mathrm{NiTi}$ conseguem retornar à sua forma originalmente definida quando deformadas em até $8 \%{ }^{5}$.

Entretanto, os arcos de NiTi apresentam baixa resistência à corrosão diante dos íons fluoreto, aumentando a rugosidade de superfície ${ }^{6}$, afetando o atrito do conjunto arco-braquete, influenciando na eficácia do tratamento, além de também poder iniciar reações tóxicas e alérgicas pela liberação do níquel ${ }^{7,8}$. O flúor pode estar presente na água, em medicamentos ou em alimentos. Peixe de água salgada têm níveis relativamente elevados de fluoreto (1 a 3mg / 100g), bem como no chá (aproximadamente 0,5 a 1,5mg/l). Em produtos médicos (pasta de dentes, enxaguatórios bucais e flúor), o flúor pode variar entre $0,1 \%$ (1000 ppm) e 1\% (10.000 ppm) e pode até mesmo chegar a $2 \%(20.000$ ppm) em aplicações profissionais ${ }^{9}$.

A dificuldade de higienização dos pacientes em tratamento ortodôntico, resulta no acúmulo de placa ao redor dos braquetes, podendo levar ao surgimento de lesões brancas. Para esses casos, é frequente a prescrição de soluções fluoretadas pelo ortodontista, a fim de aumentar a capacidade de remineralização do esmalte. Os dentifrícios e enxaguatórios comuns apresentam um bom efeito na prevenção da desmineralização, no entanto, são as soluções com altas concentrações de flúor que produzem melhores resultados de remineralização ${ }^{10}$.

Os íons fluoreto degradam a camada passiva do óxido de titânio da superfície dos arcos de NiTi, através da absorção de hidrogénio ${ }^{11}, 12$, reduzindo a sua resistên- cia à corrosão, influenciando, assim, na biocompatibilidade ${ }^{6}$. Fatores como tempo de exposição, concentração de flúor e um pH mais ácido podem facilitar a corrosão da superfície de ligas de $\mathrm{NiTi}$, uma vez que esses fatores estão interligados ${ }^{12-14}$. É evidente que a corrosão do titânio acontece não só pela concentração do flúor, mas também pelo $\mathrm{pH}^{15}$. Para degradar a camada passiva dos arcos NiTi é necessária a concentração crítica de 30 ppm de flúor $^{16}$. Isso ocorre em solução de 500 ppm de flúor a $\mathrm{pH}=4^{15}$. O titânio não foi considerado ser resistente em solução contendo 500 ppm de flúor, com pH = 4 ou solução neutra com 2250 ppm ${ }^{11}$.

Apesar disso, ainda não há um consenso de qual seria o $\mathrm{pH}$ crítico necessário para levar a corrosão dos arcos $\mathrm{NiTi}$ em soluções de flúor a 1,1 \% em soluções para bochecho, imaginando que, em média, um arco permanece na cavidade bucal por volta de 90 dias. Nesse intuito, o objetivo deste estudo foi avaliar qual seria o pH limítrofe para ocorrer a corrosão de arcos NiTi em soluções de flúor a 1,1 \%, variando o $\mathrm{pH}$ de 3,5 a 7,0.

\section{MATERIAIS E MÉTODOS}

\section{Delineamento Experimental}

O fator avaliado neste estudo foi desafio à solução de flúor a $1,1 \%$ em 8 níveis de $\mathrm{pH}(3,5 ; 4,0 ; 4,5 ; 5,0 ; 5,5 ; 6,0 ; 6,5$ e $7,0)$ além do controle positivo (água). Formando ao final 9 grupos, com duas repetições para cada grupo. A variável de resposta foi qualitativa, através de imagens obtidas por microscopia eletrônica de varredura (MEV).

\section{Obtenção das amostras}

As 18 amostras foram obtidas de arcos superiores de $\mathrm{NiTi}$ superelástico 0017 x 0025 (Abzil, São José do Rio Preto, Brasil; lote: 0934100225), com 10mm de comprimento, obtidas do segmento distal dos arcos.

\section{Desafio das amostras}

A cada duas amostras, foram aleatoriamente separadas e imersas dentro de recipientes contendo $40 \mathrm{ml}$ das seis soluções (água, soluções de flúor com pH: 3,5; 4,0; $4,5 ; 5,0 ; 5,5 ; 6,0 ; 6,5$ e 7,0$)$, sob uma mesa agitadora (Nova Ética Produtos e Equipamentos Científicos Ltda, São Paulo, Brasil) 


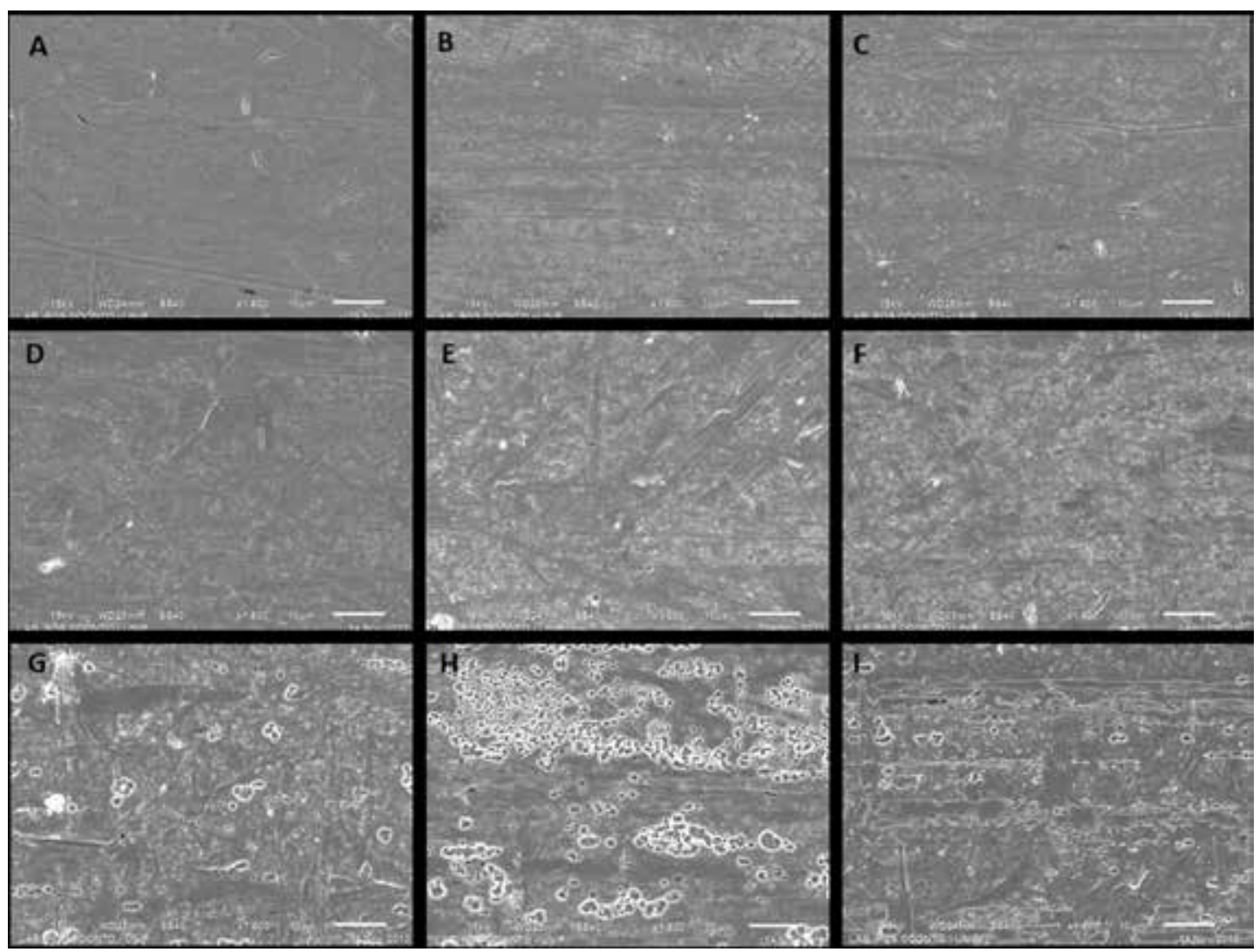

Figura 1. Imagens obtidas pelo MEV com aumento de 400x após a imersão em água e soluções de flúor com diferentes valores de $\mathrm{pH}$ : A: Controle água; $B: 7,0 ; C: 6,5 ; D: 6,0$; E:5,5; F: 5, O; G:4,5; H:4,0; I:3,5.

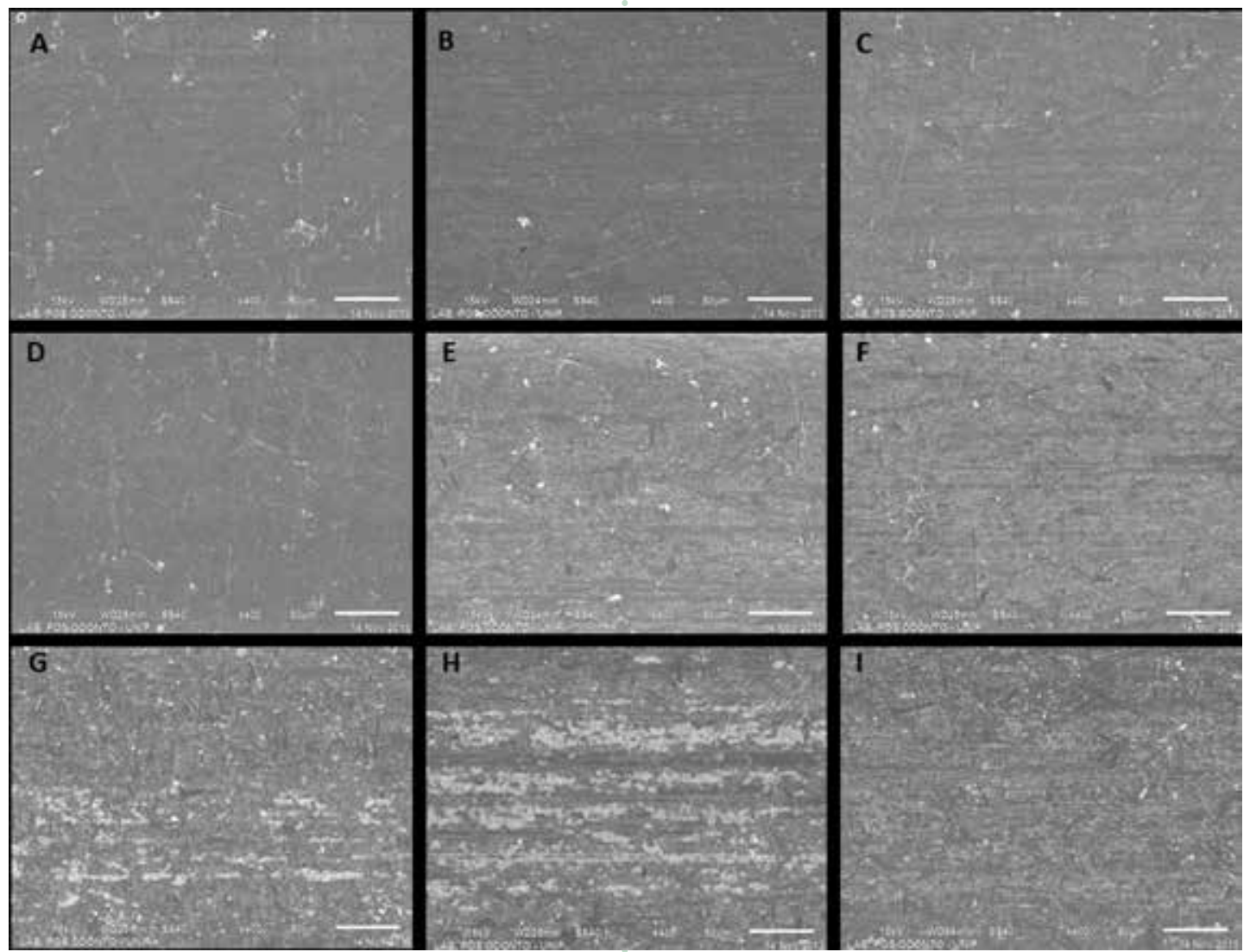

Figura 2. Imagens obtidas pelo MEV com aumento de 1600x após a imersão em água e soluções de flúor com diferentes valores de $\mathrm{pH}$ : A: Controle água; $B: 7,0 ; C: 6,5 ; D: 6,0$; E:5,5; F: 5, O; G:4,5; H:4,0; I:3,5.

MAEDA FA AMARAL N OGAWA CM

FARIAS SA

FERNANDES KS

CARVALHO PEG

TRIVIÑO T

SILVA LH

O EFEITO DE DIFERENTES

NÍVEIS DE PH EM SOLUÇÕES DE FLÚOR a 1,1\% NA corrosão de ARCOS NITI

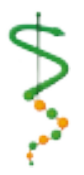


MAEDA FA

AMARAL N

OGAWA CM

FARIAS SA

FERNANDES KS

CARVALHO PEG

TRIVIÑO T

SILVA LH

O EFEITO DE

DIFERENTES

NÍVEIS DE PH

EM SOLUÇÕES DE

FLUUOR A $1,1 \%$

NA CORROSÃO DE ARCOS NITI

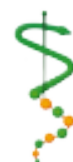

REV, ODONTOL

UNIV, CID, SÃO

PAULO

2017; 29(3): $237-42$, SET - DEZ
I SSN 1983-5183

a 66 ciclos/min, durante 90 minutos.

Preparo das amostras para MEV

Após o desafio, todas as amostras foram enxaguadas em água deionizada por 30s, e, em seguida, imersas em ultrassom (Unique - Ultra cleaner 1.400) em recipientes contendo acetona P.A. e posteriormente álcool isopropílico, por $5 \mathrm{~min}$ cada imersão, secas então em papel absorvente e com ar comprimido. Todas as amostras foram, então, levadas ao microscópio eletrônico de varredura (MEV - JEOL. JSM 7401 F, Tóquio, Japão).

\section{RESULTADOS}

As imagens geradas pelo MEV (figuras 1 e 2) demonstraram que em 90 minutos de imersão das amostras em soluções fluoretadas com pH 7,0 (1B, 2B), 6,5 (1C, $2 C)$ e $6,0(1 D, 2$ D) não se diferenciaram da imersa em água deionizada. Porém, as amostras imersas em soluções fluoretadas com pH 5,5 (1E, 2E) e 5,0 (1F, 2F) apresentaram imagens de superfície com características diferentes das amostras anteriores, sugerindo corrosão. Já as amostras imersas em soluções fluoretadas com $\mathrm{pH}$ menores, $4,5(1 \mathrm{G}, 2 \mathrm{G}), 4,0(1 \mathrm{H}, 2 \mathrm{H})$ e 3,5 $(1 \mathrm{I}, 2 \mathrm{I})$, apresentaram características ainda mais sugestivas de corrosão comparando-se com as imagens anteriores.

\section{I SCUSSÃO}

Durante o tratamento ortodôntico, pacientes que fazem uso de aparelhos fixos frequentemente apresentam a higiene bucal comprometida, o que pode dificultar até mesmo o tratamento com o surgimento de áreas de esmalte desmineralizadas e cárie $^{17}$. Para combater esse potencial problema, os ortodontistas comumente prescrevem soluções fluoretadas para uso diário10. Alguns profissionais preferem a prescrição de soluções fluoretadas aciduladas por apresentarem uma dupla ação; a primeira seria fornecer íons de cálcio para a interação com os íons de fluoreto; e a segunda seria reduzir a quantidade de $\mathrm{HPO}_{4}^{2}$ - disponível, inibindo, assim, a deposição de fluoreto de cálcio, levando à formação de um produto mais facilmente solúvel ${ }^{18}$.

As soluções fluoretadas utilizadas neste estudo foram manipuladas com a concen- tração de 1,1\%, baseada no estudo de Walker et al. ${ }^{13}$. Estes autores justificam as escolhas por essa mesma concentração estar presente em dois produtos amplamente utilizados: Phos-flur gel e Prevident 5000 (ambos produtos da Colgate Palmolive), produtos que só apresentam diferenças no $\mathrm{pH}, 5,1$ e 7,0 respectivamente. $\mathrm{O}$ tempo de imersão de 90 min também foi baseado nesse mesmo trabalho, pois equivaleria a 3 meses com o uso diário por 1 minuto que poderia ser em solução de colutório ou em gel.

Neste trabalho, as imagens obtidas dos arcos de NiTi, que foram imersos em soluções fluoretadas com $\mathrm{pH}$ entre 7,0 e 6,0 , não se mostraram muito diferentes das imagens do grupo-controle, achados semelhantes aos relatados por Koushik et al. ${ }^{19}$. Entretanto, são diferentes das imagens encontradas por Mane et al. ${ }^{20}$, que relataram que, utilizando uma solução fluoretada semelhante à deste estudo, 1,1\% com pH 7,0 imersa por 90 min, exibiram alterações corrosivas superficiais. Yadav et al. ${ }^{21}$ também encontraram a corrosão superficial dos arcos NiTi em soluções fluoretadas com $\mathrm{pH} 6,54$, porém a concentração era de $0,05 \%$ de fluoreto, somado a isso, essa solução também afetaria negativamente na força de deflexão com dispositivo de 3 pontos quando comparado à água deionizada.

As amostras imersas em soluções fluoretadas com pH 5,5 e 5,0 apresentaram imagens de superfície com características diferentes das amostras com $\mathrm{pH}$ mais básicos, apresentando alterações que poderiam possivelmente ser associadas à corrosão superficial, resultados estes que corroboram outros estudos que utilizaram o Phos-Flur e apresentaram imagens com características semelhantes ${ }^{9,19-21}$. Já as amostras imersas em soluções fluoretadas com pH menores, 4,5 a 3,5, apresentaram características ainda mais sugestivas de corrosão, concordando com os achados de Rerhrhaye et al. ${ }^{9}$ e Ramalingam et al. ${ }^{22}$. O comportamento de corrosão de titânio seria afetado não só pela concentração de flúor, mas também por $\mathrm{pH}^{15,21}$. Segundo o trabalho clínico de Ramalingam et al. ${ }^{22}$, que utilizaram duas soluções ácidas de flúor, o grupo mais ácido apresentou alte- 
rações no módulo de elasticidade.

Apesar de ser evidente que o flúor é benéfico na prevenção da cárie, o ortodontista deve estar ciente de que esse material preventivo pode vir a afetar o tratamento ortodôntico. A liberação de substâncias tóxicas, especialmente $\mathrm{Ni}$, poderia ser responsável por alergias em indivíduos predispostos $^{23}$. As ranhuras na superfície dos arcos $\mathrm{NiTi}$, resultantes da corrosão, podem aumentar o atrito, retardando o alinhamento dental durante a fase de nivelamento ${ }^{24}$, e poderiam dar origem à fratura dos arcos NiTi na presença de um defeito estrutural decorrente de algum problema durante o processo de fabricação.

\section{CONCLUSÃO}

Com base nos resultados obtidos neste estudo, o pH crítico para corrosão de arcos NiTi em soluções de flúor a 1,1\% parece estar entre 6,0 e 5,5. Uma boa higiene oral deve ser ensinada e cobrada a cada consulta, a fim de promover a eliminação mecânica da placa bacteriana. Porém, se ainda assim o ortodontista perceber que o paciente possui risco de cáries, deve-se optar pela prescrição de soluções fluoretadas com pH mais básico.

\section{REFERÊNCIAS}

1. Schiff $N$, Boinet $M$, Morgon L, Lissac M, Dalard F, Grosgogeat B. Galvanic corrosion between orthodontic wires and brackets in fluoride mouthwashes. European journal of orthodontics. 2006;28(3):298-304.

2. Persson M. A 100th anniversary: Sandstedt's experiments on tissue changes during tooth movement. Journal of orthodontics. 2005;32(1):27-8.

3. Sandhu SS, Shetty VS, Mogra S, Varghese J, Sandhu J, Sandhu JS. Efficiency, behavior, and clinical properties of superelastic $\mathrm{NiTi}$ versus multistranded stainless steel wires: a prospective clinical trial. The Angle orthodontist. 2012;82(5):915-21.

4. Kusy RP. A review of contemporary archwires: their properties and characteristics. The Angle orthodontist. 1997;67(3):197-207.

5. Dayananda GN, Subba Rao M. Effect of strain rate on properties of superelastic NiTi thin wires. Mater Sci Eng $A$ Struct Mater. 2008;486(1-2):8.

6. Huang $\mathrm{HH}$. Effects of fluoride concentration and elastic tensile strain on the corrosion resistance of commercially pure titanium. Biomaterials. 2002;23(1):5.
7. Kao CT, Ding SJ, He H, Chou MY, Huang TH. Cytotoxicity of orthodontic wire corroded in fluoride solution in vitro. The Angle orthodontist. 2007;77(2):349-54.

8. Kassab EJ, Gomes JP. Assessment of nickel titanium and beta titanium corrosion resistance behavior in fluoride and chloride environments. The Angle orthodontist. 2013;83(5):864-9.

9. Rerhrhaye W, Bahije L, El Mabrouk K, Zaoui F, Marzouk N. Degradation of the mechanical properties of orthodontic NiTi alloys in the oral environment: an in vitro study. International orthodontics / Collège européen d'orthodontie. 2014;12(3):271-80.

10. Alexander SA, Ripa LW. Effects of self-applied topical fluoride preparations in orthodontic patients. The Angle orthodontist. 2000;70(6):424-30.

11. Lee TH, Huang TK, Lin SY, Chen LK, Chou MY, Huang $\mathrm{HH}$. Corrosion resistance of different nickel-titanium archwires in acidic fluoride-containing artificial saliva. The Angle orthodontist. 2010;80(3):547-53.

12. Yokoyama K, Kaneko K, Moriyama K, Asaoka K, Sakai J, Nagumo M. Hydrogen embrittlement of $\mathrm{Ni}-\mathrm{Ti}$ superelastic alloy in fluoride solution. J Biomed Mater Res A. 2003;652(2):6.
MAEDA FA

AMARAL N

OGAWA CM

FARIAS SA

FERNANDES KS

CARVALHO PEG

triviño t

SILVA LH

O EFEITO DE DIFERENTES

NÍVEIS DE PH

EM SOLUÇÕES DE

FLÚOR A 1,1\%

NA CORROSÃO DE

ARCOS NITI

241
REV, ODONTOL.

UNIV. CID. São

PAULO

2017; 29(3):

$237-42$, SET-DEZ 
MAEDA FA

AMARAL N

OGAWA CM

FARIAS SA

FERNANDES KS

CARVALHO PEG

TRIVIÑO T

SILVA LH

O EFEITO DE

DIFERENTES

NIVVEIS DE PH

EM SOLUÇÕES DE

FLUUOR A $1,1 \%$

NA CORROSÃO DE

ARCOS NITI

242
13. Walker MP, White RJ, Kula KS. Effect of fluoride prophylactic agents on the mechanical properties of nickel-titanium-based orthodontic wires. American journal of orthodontics and dentofacial orthopedics. 2005;127(6):662-9.

14. Watanabe I, Watanabe E. Surface changes induced by fluoride prophylactic agents on titanium-based orthodontic wires. American journal of orthodontics and dentofacial orthopedics. 2003;123(6):653-6.

15. Nakagawa M, Matsuya S, Shiraishi T, Ohta M. Effect of fluoride concentration and $\mathrm{pH}$ on corrosion behavior of titanium for dental use. Journal of dental research. 1999;78(9):1568-72.

16. Lausmaa J, Kasemo B, Hansson S. Accelerated oxide grown on titanium implants during autoclaving caused by fluorine contamination. Biomaterials. 1985;6(1):5.

17. Gorelick L, Geiger AM, Gwinnett AJ. Incidence of white spot formation after bonding and banding. American journal of orthodontics. 1982;81(2):93-8.

18. Rolla G, Saxegaard E. Critical evaluation of the composition and use of topical fluorides, with emphasis on the role of calcium fluoride in caries inhibition. Journal of dental research. 1990;69 Spec No:780-5; discussion 820-3.
19. Koushik SRH, Hegde N, Mahesh CM, Chandrashekar BS, Shetty B, Mahendra S. Effect of fluoride prophylactic agents on the mechanical properties of Nickel-Titanium Wires: An in vitro Study. J Ind Orthod Soc. 2011;45(4):6.

20. Mane PP, Pawar R, Ganiger C, Phaphe S. Effect of fluoride prophylactic agents on the surface topography of $\mathrm{NiTi}$ and CuNiTi wires. The journal of contemporary dental practice. 2012;13(3):285-8.

21. Yadav SK, Sehgal V, Mittal S. Effects of Fluoride on NiTi Orthodontic Archwires: An X-ray Diffraction Study. J Ind Orthod Soc. 2013;47(4):5.

22. Ramalingam A, Kailasam V, Padmanabhan S, Chitharanjan A. The effect of topical fluoride agents on the physical and mechanical propertiesofNiTiandcopper $\mathrm{NiTi}$ archwires. An in vivo study. Australianorthodonticjournal.2008;24(1):2631.

23. Bogdanski D, Koller M, Muller D, Muhr G, Bram M, Buchkremer HP, et al. Easy assessment of the biocompatibility of $\mathrm{Ni}-\mathrm{Ti}$ alloys by in vitro cell culture experiments on a functionally graded Ni-NiTi-Ti material. Biomaterials. 2002;23(23):4549-55.

24. Jaber LC, Rodrigues JA, Amaral FL, Franca FM, Basting RT, Turssi CP. Degradation of orthodontic wires under simulated cariogenic and erosive conditions. Brazilian oral research. 2014;28(1).

Recebido em 14/08/2017

Aceito em 16/10/2017
REV, ODONTOL.

UNIV. CID. SÃO PAULO:

$2017 ; 29(3)$ : 237-42, SET-DEZ 